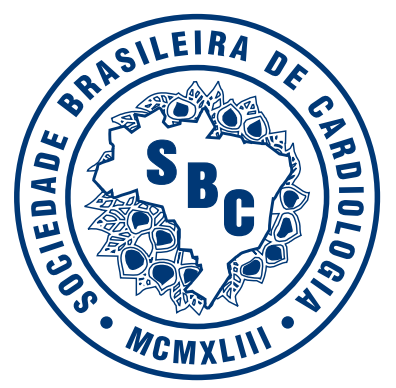

\title{
I DIRETRIZ DE RESSONÂNCIA E TOMOGRAFIA CARDIOVASCULAR DA SOCIEDADE BRASILEIRA DE CARDIOLOGIA - SUMÁRIO EXECUTIVO
}

\section{Realização}

Grupo de Estudos de Ressonância e Tomografia Cardiovascular (GERT) do Departamento de Cardiologia Clínica da Sociedade Brasileira de Cardiologia

EDITOR

Carlos Eduardo Rochitte

Co-Editor

Ibraim Masciarelli Francisco Pinto

\section{Editores Associados}

Juliano Lara Fernandes, Clério Francisco Azevedo Filho, Adib Jatene, Antonio Carlos de Camargo Carvalho, Jorge Pinto Ribeiro, José Antonio Franchini Ramires, Sergio Almeida Oliveira

\section{Colaboradores}

César Augusto Mastrofrancisco Cattani, Dany Jasinowodolinsk, Fabiano Lucchesi, Fábio Berezowsky Rocha, Fátima Cristina Pedroti, Gilberto Szarf, Guilherme Urpia Monte, lugiro Roberto Kuroki , Joalbo Andrade, José Rodrigues Parga Filho, Luis Cláudio Correia, Luiz Francisco Ávila, Marcelo Hadlich, Marcelo Zapparoli, Marcia Barbosa, Márcia Lima Mugnaini, Maria Helena Albernaz Siqueira, Marly Maria Uellendhal, Miguel Abraão Rosário Neto, Paulo R. Schwarzman, Raul Dias dos Santos Filho, Ricardo Loureiro, Roberto Kalil Filho, Robson de Macedo Vieira

Correspondência: Carlos Eduardo Rochitte • Instituto do Coração (InCor) HC - FMuSP - Av. Dr. Enéas de Carvalho Aguiar 44 - 05403-000 - São Paulo- SP 


\section{INTRODUÇÃO}

O uso da ressonância magnética e da tomografia computadorizada no diagnóstico das doenças cardiovasculares apresentou desenvolvimento tecnológico expressivo recente, o que levou a um aumento na utilização destas tecnologias por parte de cardiologistas e médicos envolvidos no manejo de pacientes cardiopatas. Apesar disso, orientações específicas quanto ao seu uso ainda são escassas, limitando-se a Task Force da American Heart Association (AHA) e American College of Cardiology (ACC) sobre a tomografia computadorizada cardiovascular (TCC) por feixe de elétrons na abordagem do escore de cálcio ${ }^{1}$ e pelo Consensus Panel Report envolvendo especialistas europeus sobre a utilização da ressonância magnética cardiovascular (RMC) ${ }^{2}$. Contudo, diretrizes formais da ACC ou AHA ainda não foram publicadas para nenhuma das duas modalidades.

Assim, o grupo de estudos em ressonância e tomografia cardiovascular (GERT) da SBC elaborou estas diretrizes baseado em inúmeros trabalhos publicados na área, experiência de especialistas e opinião de cardiologistas de grande reconhecimento científico e que utilizam os métodos com freqüência. O uso diagnóstico da RMC e da TCC é aqui descrito com foco no cardiologista clínico, no contexto de outras modalidades de imagens, levando-se em conta, inclusive, relações de custo-efetividade.

\section{Classificação}

Baseado nos aspectos de medicina baseada em evidência ${ }^{3}$ e como não existe, ainda, uma classificação dos níveis de indicação para a RMC e TCC ou grandes estudos multicêntricos e randomizados ${ }^{3}$, optou-se por utilizar a seguinte classificação:

Classe I: fornece informações relevantes e, geralmente, é apropriada; pode ser usada como técnica de imagem de primeira linha; geralmente substanciada por estudos que avaliam sensibilidade, especificidade, valores preditivos positivo e negativo e acurácia do método em relação a métodos diagnósticos de referência com literatura consistente e abundante e/ou opiniões concordantes.

Classe II: condições nas quais há evidência conflitante e/ou divergência de opiniões sobre a utilidade/eficácia de determinado procedimento:

Ila: O nível de evidência/opinião é a favor de sua utilização/eficácia.
Ilb: A utilização/eficácia é menos estabelecida por evidência/opinião.

Classe III: Situações onde há consenso de que o procedimento não é útil/eficaz e, em alguns casos, os riscos são maiores que os benefícios.

\section{Ressonância Magnética Cardiovascular (RMC)}

\section{TéCnicas de RessonânCia Magnética}

A ressonância magnética utiliza ondas de radiofreqüência para adquirir informações a partir dos íons de hidrogênio, não utilizando radiação ionizante. Uma vez dentro do aparelho de ressonância, os núcleos dos átomos de hidrogênio se alinham em relação ao campo magnético gerado e são estimulados temporariamente por ondas de radiofreqüência, modificando seu vetor de magnetização e refletindo a energia recebida. Esta é captada por antenas de radiofreqüência e transformada em sinais que compõem a imagem. Dependendo da programação destas ondas, denominadas seqüências de pulso, formam-se imagens utilizadas para a avaliação completa cardiovascular: avaliação anatômica, em cine para avaliação de função ventricular, detecção de infarto e viabilidade, pesquisa de isquemia, análises de fluxo ou angiografias. Diferentemente de outros exames convencionais de ressonância, a participação do médico na preparação do paciente, na aquisição das imagens e no pós-processamento é mais intensa.

Os estudos de RMC são realizados em apnéia expiratória, em geral facilmente tolerados pelos pacientes, sendo cada vez mais curtas devido à melhora da tecnologia (podendo ser tão curtas como 5 segundos). As principais contra-indicações verdadeiras e algumas situações que não são contra-indicações reais, mas que podem gerar dúvida, estão listadas na Tabela 1. Claustrofobia pode ser um problema para uma pequena parcela dos pacientes (< $2.0 \%$ em estudos clínicos), mas usualmente é contornado com orientações pré-procedimento ou medidas simples como a utilização de ansiolíticos. Deve-se ressaltar que stents coronários, embora tenham nas recomendações dos fabricantes como contra-indicação para RMC nas primeiras semanas após implante (fato que ocorre devido à regulamentação existente na época em que foram

\section{Tabela 1 - Contra-indicações e dúvidas comuns em cardiologia para exames de RMC}

$\begin{array}{ll}\text { Não podem realizar exames } & \text { Podem realizar exames } \\ \text { Portadores de marcapasso } & \text { Pacientes com stents coronários (mesmo na fase aguda) } \\ \text { Portadores de desfibriladores implantados } & \text { Portadores de valvas artificiais (biológicas ou metálicas) } \\ \text { Pacientes com clipes cerebrais } & \text { Pacientes submetidos a cirurgias cardíacas com sutura de esterno } \\ \text { Implantes cocleares } & \text { Pacientes com próteses de aorta } \\ \text { Fragmentos metálicos nos olhos } & \\ \text { * uma lista completa de contra-indicações e permissões pode ser encontrado no endereço www.mrisafety.com }\end{array}$

\section{Palavras-chave}

Imagem por ressonância magnética, tomografia, técnicas de diagnóstico cardiovascular 
lançados no mercado), não representam risco para o paciente mesmo imediatamente após o implante ${ }^{4}$.

Para realização da maioria dos exames de RMC e de praticamente todos os exames de angiografia por ressonância, é utilizado um contraste endovenoso baseado no elemento paramagnético gadolínio $(\mathrm{Gd})^{5}$. Várias moléculas estão disponíveis comercialmente, sendo que todas apresentam distribuição extra-celular, isto é, se distribuem no espaço vascular e intersticial, não penetrando no interior de células com membrana celular íntegra. Esta característica permite que estes contrastes sejam usados para a análise da perfusão tecidual, identificação de trombos, massas neoplásicas, áreas de fibrose ou infarto e alterações estruturais do miocárdio. Os efeitos adversos dos contrastes baseados em Gd (cefaléia, náuseas ou alterações de paladar) são bastante raros, com efeitos graves inferiores a 0,01\% (1:10.000) dos exames ${ }^{6}$, não apresentando também, nas doses clinicamente utilizadas, nefrotoxicidade ou hepatotoxicidade.

\section{INDICAÇÕES DE RMC}

\subsection{Cardiopatias Congênitas}

A RMC é considerada uma técnica de imagem complementar à ecocardiografia, fornecendo informação morfofuncional incremental com impacto diagnóstico e terapêutico em pacientes selecionados ${ }^{7}$, sobretudo em casos de crianças maiores ou adultos, em anatomias complexas e após intervenções cirúrgicas ${ }^{8}$. A RMC pode ser empregada nas seguintes anomalias congênitas (Tabela 2):

1. Anomalias de situs víscero-atrial. A RMC fornece informação anatômica ampla, incluindo estruturas extracardíacas $^{9}$, tornando-se um método diagnóstico com sensibilidade próxima a $100 \%{ }^{10}$.

2. Anomalias atriais e venosas. Em situações onde se deseja avaliar anomalias do retorno venoso, como na drenagem venosa parcial anômala ${ }^{11}$ ou na avaliação pós-operatória de estenoses ou oclusões das veias pulmonares $^{12}$.

3. Anomalias dos ventrículos. Especialmente em cardiopatias complexas, como na Tetralogia de Fallot, atresia pulmonar, atresia tricúspide e corações univentriculares ${ }^{10}$, a RMC avalia morfologia, volumes e massas ventriculares, sendo opção ao ecocardiograma em defeitos isolados do septo interventricular.

4. Valvas. A principal indicação da RMC nestas situações está na avaliação de estenoses aórticas supravalvares e na determinação da regurgitação pulmonar.

5. Anomalias dos vasos da base e tubos. A RMC pode ser o exame de escolha na avaliação da coarctação da aorta, de anéis vasculares e na avaliação da aorta após intervenção cirúrgica ou percutânea ${ }^{13}$.

6. Pós-operatório de cardiopatias congênitas. A avaliação seriada do ventrículo direito é de valor no manuseio pós-cirúrgico de pacientes com sobrecarga desta câmara ${ }^{14}$.

7. Anomalias das artérias coronárias. A RMC é útil na caracterização de anormalidades congênitas e adquiridas, como na coronária esquerda originando-se do tronco pulmonar e na doença de Kawasaki com aneurismas coronários $^{15}$.

\section{Tabela 2 - Indicações de RMC nas Cardiopatias} Congênitas

Indicação Classe

Indicações gerais

1. Seguimento de cardiopatias congênitas do adulto Indicações específicas

1. Avaliação de shunt sistêmico-pulmonar (Qp/Qs)

2. Anomalias de situs víscero-atrial

Anomalias de situs com cardiopatias congênitas complexas

Anomalias isoladas de situs

3. Anomalia atrial e do retorno venoso

Retorno venoso pulmonar anômalo, especialmente em anomalias complexas e cor triatriatum

Retorno venoso sistêmico anômalo Obstrução de retorno venoso pulmonar ou sistêmico após reparo intra-atrial ou correção de retorno venoso pulmonar anômalo

Comunicação inter-atrial isolada (secundum e primum)

4. Anomalias das valvas átrio-ventriculares

Anomalia de Ebstein

lasse

Anomalias anatômicas das valvas mitral e tricúspide

Anomalias valvares funcionais

Defeito do septo átrio-ventricular isolado

5. Anomalias dos ventrículos

Comunicação interventricular associada com anomalias complexas

Comunicação interventricular supra-cristal

Avaliação da função ventricular, massa e volumes

direito e esquerdo

Aneurismas e divertículos ventriculares

Comunicação interventricular isolada

6. Anomalias das valvas semilunares

Estenose aórtica supra-valvar

Regurgitação pulmonar

Estenose pulmonar supra-valvar

Estenose valvar aórtica isolada

Estenose aórtica sub-valvar

Valva aórtica bicúspide

Displasia e estenose valvar pulmonar isolada

7. Anomalias das artérias

Avaliação pós-operatória de shunts

Aneurismas do seio de Valsalva

Coarctação da aorta

Anéis vasculares

Janela aorto-pulmonar

Origem anômala de coronárias em adultos e crianças maiores

Atresia pulmonar

Estenose pulmonar proximal

Colaterais sistêmico-pulmonares

Má-posição dos grandes vasos

Canal arterial persistente
I

III

IIb

III

III 


\subsection{Doenças Vasculares - RMC}

A RMC, especialmente pela técnica de angiografia por ressonância (angio-RMC) com uso do contraste baseado em gadolínio, tem grande utilidade na avaliação de diversas doenças vasculares, permitindo a aquisição em três dimensões e liberdade de escolha de planos ${ }^{16}$. Além disso, também permite a caracterização da parede vascular, trombos e análise quantitativa de fluxos ${ }^{17}$. As principais indicações da RMC para avaliação das doenças vasculares estão na Tabela 3.

\begin{tabular}{|c|c|}
\hline Indicação & Classe \\
\hline 1. Aneurismas de aorta (incluindo Marfan) & 1 \\
\hline 2. Dissecção de aorta & I \\
\hline 3. Ruptura de aorta & I \\
\hline 4. Hematoma intramural aórtico & I \\
\hline 5. Úlceras aórticas & I \\
\hline 6. Planejamento de abordagem cirúrgica da aorta & I \\
\hline 7. Planejamento de stent aórtico & । \\
\hline 8. Arterites & I \\
\hline 9. Anatomia da artéria pulmonar e fluxo & I \\
\hline 10. Avaliação das veias pulmonares & I \\
\hline 11. Avaliação estenoses renais & । \\
\hline 12. Avaliação de estenoses carótidas extra-cranianas & I \\
\hline 13. Embolia pulmonar & $\mathrm{IIb}$ \\
\hline
\end{tabular}

A RMC pode ser utilizada nas principais doenças da aorta e de seus ramos principais, na avaliação das veias pulmonares pré ou pós ablação da fibrilação atrial, no diagnóstico de obstruções de artérias carótidas e na avaliação de estenoses de artérias renais em casos de hipertensão renovascular ou em pacientes nefropatas. Apesar da menor resolução espacial em relação à angiografia convencional ou a angiotomografia, a RMC pode fornecer dados quanto à importância de lesões estenóticas de maneira reprodutível e com alta sensibilidade e especificidade ${ }^{18}$, não utilizando contrastes nefrotóxicos, sendo a técnica de escolha na avaliação das artérias renais de pacientes com função renal alterada.

\subsection{Doença Arterial Coronária}

\section{Introdução}

A RMC na DAC agrega informações únicas, integrando diversas respostas clínicas num mesmo exame ${ }^{19}$. As indicações para a RMC na avaliação da doença arterial coronária estão sintetizadas na tabela 4.

\section{Função, massa, volumes e geometria ventricular}

A avaliação da morfologia e função do ventrículo
Tabela 4 - Indicações de RMC na avaliação da doença arterial coronária

$\begin{array}{lc}\text { Indicação } & \text { Classe } \\ \text { 1. Avaliação da função ventricular global, volumes e } & \text { I } \\ \text { massa (esquerda e direita) } & \\ \text { 2. Detecção isquemia miocárdica } & \\ \text { Avaliação da função ventricular regional em repouso e } & \text { Ila } \\ \text { em estress (RMC-estresse) } & \text { Ila } \\ \text { Avaliação da perfusão miocárdica } & \\ \text { 3. Infarto agudo e crônico do miocárdio } & \text { I } \\ \text { Detecção e quantificação } & \text { I } \\ \text { Viabilidade miocárdica } & \text { I } \\ \text { Trombo ventricular } & \text { I } \\ \text { Avaliação de aneurisma de VE } & \text { Ila } \\ \text { Diagnóstico de síndrome coronária na fase aguda } & \text { III } \\ \text { Comunicação interventricular } & \text { III } \\ \text { Insuficiência mitral aguda } & \\ \text { 4. Angio-RMC de artérias coronárias } & \text { I } \\ \text { Anomalias congênitas } & \text { III } \\ \text { Doença arterial coronária } & \text { III } \\ \text { Avaliação de patência de enxertos }\end{array}$

validada fazem da RMC método padrão de referência para análise de parâmetros geométricos cardíacos e para a análise seriada ao longo do tempo de suas modificações. Sua reprodutibilidade tem sido relatada como superior ao ecocardiograma e à cintilografia miocárdica perfusional tomográfica engatilhada (gated SPECT) ${ }^{20}$. Assim, a RMC permite avaliação acurada e reprodutível da disfunção global e segmentar do ventrículo esquerdo e do ventrículo direito, mesmo em pacientes com alterações significativas da geometria ventricular (como no caso de aneurismas ventriculares ou remodelamento importante).

\section{Detecção de isquemia - RMC de estresse}

A RMC de estresse com dobutamina utiliza a avaliação da contração regional do VE em repouso e durante infusão de dobutamina, semelhante à ecocardiografia com dobutamina. Este exame tem alta acurácia, com resultados relatados como superiores ao ecocardiograma de estresse ${ }^{21}$. 0 exame apresenta importante valor prognóstico ${ }^{22}$, podendo ser utilizado na avaliação do risco pré-operatório de cirurgia não cardíaca ${ }^{23}$.

\section{Detecção da isquemia - perfusão miocárdica}

A pesquisa de isquemia pela avaliação da perfusão miocárdica utiliza na rotina clínica a avaliação qualitativa da passagem do contraste pelo miocárdio após sua injeção em veia periférica ${ }^{24}$. Obtém-se imagens na fase de estresse induzido com dipiridamol ou adenosina e na fase de repouso, com boa acurácia diagnóstica em relação à cinecoronariografia invasiva, à tomografia de emissão de pósitrons (PET) e à cintilografia miocárdica perfusional $(\mathrm{SPECT})^{25}$. Há relatos de resultados superiores da RMC 
em comparação à cintilografia miocárdica perfusional, sobretudo no que diz respeito à sensibilidade ${ }^{26}$. Como outros métodos de indução e detecção de isquemia, a RMC também agrega valor prognóstico, mostrando baixo índice de eventos em um ano nos pacientes com perfusão normal ${ }^{27}$.

\section{Detecção do infarto do miocárdio}

A RMC baseada na técnica do realce tardio miocárdico permite delimitação das áreas de necrose ou fibrose miocárdica nos pacientes com infarto prévio ${ }^{28}$. A técnica baseia-se na propriedade dos contrastes baseados no gadolínio em se distribuir de forma diferenciada entre o tecido normal e o tecido infartado, tendo uma saída mais lenta deste último, gerando uma diferença de sinal de até $1080 \%$ entre os dois tecidos. Assim, áreas de infarto são identificadas de maneira precisa, com alta acurácia e reprodutibilidade. A técnica permite, inclusive, a detecção de infartos de pequenas dimensões, não identificados por outros métodos ${ }^{29}$. Além disso, a quantificação da área infartada, que pode ser obtida de maneira rápida e rotineira, tem potencial significado prognóstico ${ }^{30}$.

\section{Viabilidade miocárdica - fase aguda}

Na fase aguda, a RMC permite, através da determinação da extensão da área infartada (subendocárdica ou transmural), diferenciar áreas de miocárdio viável (atordoadas) de áreas com necrose ou fibrose sem potencial de recuperação funcional, com lesão irreversível ${ }^{31}$. A RMC também permite, ainda na fase aguda, determinar a evolução da função cardíaca, identificar o remodelamento adverso ${ }^{31}$ e determinar a extensão de áreas de obstrução microvascular ("noreflow"). A obstrução microvascular tem valor prognóstico para predizer o remodelamento adverso do ventrículo esquerdo e eventos cardiovasculares ${ }^{32}$.

\section{Viabilidade miocárdica - fase crônica}

Na fase crônica do infarto, a técnica também mais utilizada para determinação de viabilidade miocárdica é a do realce tardio. A viabilidade por RMC mostrou-se superior à cintilografia miocárdica perfusional ${ }^{29}$ e com mesma sensibilidade e especificidade a tomografia perfusional por emissão de pósitrons ${ }^{48}$. Clinicamente, baseada na identificação de segmentos do ventrículo esquerdo com áreas de realce tardio menores (viáveis) ou maiores (não viáveis) que $50 \%$ da área total do segmento, observou-se que a RMC tem valor preditivo positivo de $66-85 \%$ e valor preditivo negativo de $82-$ $92 \%$ na determinação de quais segmentos miocárdicos terão melhora funcional após a revascularização ${ }^{33}$. Devido à rapidez e facilidade na realização do exame, o método vem se tornando a primeira linha de escolha na determinação da viabilidade miocárdica na prática clínica. Um uso comum, em nosso meio, da técnica de realce tardio miocárdico é a avaliação de áreas acinéticas e discinéticas quando se suspeita de aneurisma ventricular esquerdo, em especial se o paciente tem procedimento cirúrgico de revascularização miocárdica planejado. A presença de fibrose nestes territórios reforça a indicação de aneurismectomia ou reconstrução geométrica do VE e ajuda no planejamento pré-operatório do cirurgião cardíaco.

\section{Angiografia coronária e fluxo coronário}

Apesar do avanço das técnicas de RMC para avaliação angiográfica das artérias coronárias, as dificuldades técnicas e necessidade de equipamento e software muito específicos ainda não permitem que o exame tenha uma indicação clínica de rotina ${ }^{34}$, embora em situações específicas, como na identificação das anomalias de origem e trajeto das artérias coronárias, ela possa ser usada.

\subsection{Cardiomiopatias}

A RMC tem hoje papel fundamental no diagnóstico das diversas formas de cardiomiopatias, sobretudo no que diz respeito à diferenciação diagnóstica e na informação prognóstica obtida em algumas situações. As indicações para RMC nas cardiomiopatias estão listadas na Tabela 5.

\section{Cardiomiopatia hipertrófica}

Nesta doença, a RMC adiciona ao diagnóstico convencional pelo ecocardiograma por apresentar melhor sensibilidade diagnóstica, sobretudo em situações da forma apical da doença ${ }^{35}$. Mais ainda, com a detecção de áreas de fibrose miocárdica, a RMC também permite identificar casos de maior risco de arritmias e morte súbita ${ }^{36}$.

\section{Tabela 5 - Indicações de RMC na avaliação das} cardiomiopatias

$\begin{array}{lc}\text { Indicação } & \text { Classe } \\ \text { 1. Cardiomiopatia hipertrófica } & \text { । } \\ \text { 2. Cardiomiopatia dilatada - diagnóstico diferencial com } & \text { । } \\ \text { etiologia isquêmica } & \\ \text { 3. Displasia/cardiomiopatia arritmogênica do ventrículo } & \text { । } \\ \text { direito } & \\ \text { 4. Cardiomiopatia siderótica, especialmente secundária } & \text { I } \\ \text { à talassemia } & \text { I } \\ \text { 5. Miocárdio não compactado } & \text { I } \\ \text { 6. Cardiomiopatia chagásica } & \text { Ila } \\ \text { 7. Miocardite (fase aguda ou crônica) } & \text { Ila } \\ \text { 8. Diagnóstico diferencial do coração de atleta } & \text { Ila } \\ \text { 9. Cardiomiopatia restritiva } & \text { Ila } \\ \text { 10. Sarcoidose cardíaca } & \end{array}$




\section{Hipertrofia ventricular esquerda e coração de atleta}

A RMC é hoje considerada como padrão de referência no diagnóstico e seguimento da hipertrofia ventricular esquerda ${ }^{20}$. Além disso, também tem grande utilidade na complementação diagnóstica na diferenciação de situações patológicas versus coração de atleta, através da análise da geometria ventricular e presença/ausência de fibrose miocárdica ${ }^{37}$.

\section{Cardiomiopatia dilatada}

Além da determinação de volumes e massas, a RMC adiciona informação no diagnóstico diferencial das diversas etiologias para cardiomiopatias dilatadas através do padrão de realce tardio. A localização predominantemente mesocárdica, epicárdica ou heterogênea distingue formas diversas de cardiomiopatias não isquêmicas de causas isquêmicas ${ }^{38}$.

\section{Cardiomiopatia chagásica}

Na doença de Chagas, a RMC permite a identificação precoce do envolvimento cardíaco pela doença, através da detecção de áreas de realce tardio antes da detecção por outros métodos, permitindo a estratificação mais precisa dos estágios de gravidade desta cardiomiopatia ${ }^{39}$.

\section{Displasia/cardiomiopatia arritmogênica do ventrículo direito (DCAVD)}

A RMC é considerada o melhor método diagnóstico, tanto para a avaliação da anatomia quando para a função do ventrículo direito, tendo um papel significativo no diagnóstico e no acompanhamento da DCAVD ${ }^{40}$. Embora com menor valor diagnóstico que anteriormente suspeitado, a detecção de áreas de substituição fibrogordurosa pode ter também significado clínico na doença, embora, na rotina clínica, estes achados sejam relativamente raros.

\section{Cardiomiopatia siderótica}

Em casos de sobrecarga de ferro, especialmente em pacientes com talassemia major, a RMC é hoje o principal método diagnóstico, utilizada inclusive na determinação da melhor forma terapêutica na doença, através da técnica de T2*41(lê-se: "te dois estrela").

\section{Cardiomiopatia restritiva}

Em nosso meio, as causas mais comuns desta forma de cardiomiopatia são a amiloidose e a endomiocardiofibrose. Nestas duas situações, a RMC auxilia o diagnóstico através da identificação diferenciada do sinal miocárdico, ausência de alterações pericárdicas e, sobretudo, na caracterização de realce tardio, seja ele subendocárdico associado ao preenchimento apical do ventrículo esquerdo ou ventrículo direito que caracteriza a endomiocardiofibrose ${ }^{42}$, ou difuso como na amiloidose.

\section{Miocardite}

Diversos trabalhos recentes mostram a importância da RMC no diagnóstico na fase aguda ou crônica da miocardite por técnicas de ponderação do sinal magnético e do realce tardio. 0 padrão multifocal do realce aliado ao aumento de sinal na fase aguda da doença permite uma sensibilidade $76 \%$, especificidade de $96 \%$ e acurácia de $85 \%$ no diagnóstico da doença ${ }^{43}$, sendo superior à cintilografia miocárdica com gálio e permitindo considerar o método como uma das escolhas de primeira linha na investigação desta doença ${ }^{44}$.

\section{Miocárdio não compactado}

Estudos recentes têm apontado a RMC como o método ideal para diagnóstico desta doença, dada a alta resolução espacial ${ }^{45}$. Recentemente, foi demonstrado que um índice de miocárdio não compactado sobre miocárdio compactado acima de 2,3 na diástole, determinado pela RMC, tem sensibilidade de $86 \%$ e especificidade de $99 \%$ para detecção da doença ${ }^{46}$.

\section{Sarcoidose cardíaca}

A RMC tem se mostrado de grande valor ${ }^{47}$ pela demonstração de aumento de sinal e presença do realce tardio no diagnóstico desta condição infreqüente, porém carreadora da possibilidade de morte súbita.

\subsection{Doenças do Pericárdio}

\section{Derrame pericárdico}

Em casos de derrame isolado, a RMC pode agregar valor ao ecocardiograma transtorácico, quando a janela ecocardiográfica é insuficiente ou quando o derrame apresenta morfologia e localização complexa.

\section{Pericardite constritiva}

Alterações na morfologia e na função ventricular da pericardite constritiva podem ser detectadas pela $\mathrm{RMC}$, permitindo se fazer o diagnóstico diferencial entre pericardite constritiva e cardiomiopatias restritivas ${ }^{48}$. A RMC é superior ao ecocardiograma na mensuração do espessamento pericárdico ${ }^{49}$, a principal característica da pericardite constritiva.

Tabela 6 - Indicações de RMC na avaliação de doenças pericárdicas, tumores e trombos

$\begin{array}{lc}\text { Indicação } & \text { Classe } \\ \begin{array}{l}\text { 1. Detecção e caracterização de tumores cardíacos e } \\ \text { pericardíacos }\end{array} & \text { । } \\ \begin{array}{l}\text { 2. Detecção e diagnóstico diferencial de trombos } \\ \text { ventriculares }\end{array} & \text { । } \\ \text { 3. Pericardite constritiva } & \text { Ila } \\ \text { 4. Derrame pericárdico isolado } & \text { IIb } \\ \text { 5. Detecção de trombos atriais e em apêndice atrial } & \text { III }\end{array}$




\section{Anomalias congênitas do pericárdio}

Cistos e agenesia pericárdica podem ser identificados e distinguidos de outros tumores baseando-se em suas características de intensidade de sinal e localização pela RMC.

\subsection{TUMORES E TROMBOS}

A RMC permite a determinação anatômica, extensão e relação com outras estruturas de tumores cardíacos de forma precisa ${ }^{50}$ (Tabela 6). Através de técnicas específicas, o método também fornece informações quanto à composição das massas tumorais. A caracterização tecidual de massas utiliza técnicas de perfusão, realce tardio com gadolínio, ponderações em T1 e T2 pré- e pós-contraste e saturação de gordura.

Além disso, em massas ventriculares, a RMC também permite a diferenciação de tumores com trombos cavitários com melhor sensibilidade que o ecocardiograma ${ }^{51}$. No caso de trombos atriais, o ecocardiograma é o exame de escolha, pois a RMC tem sensibilidade menor nestes casos, sobretudo na avaliação do apêndice atrial.

\subsection{Doenças Valvares}

Devido à rápida movimentação dos folhetos valvares, a RMC tem limitações no diagnóstico da maioria das doenças valvares, especialmente nas situações que envolvem avaliação da morfologia valvar ou no caso de vegetações, sendo o ecocardiograma o exame de primeira escolha nestas situações. Entretanto, a RMC pode ser útil em determinadas situações, especialmente quando se deseja avaliar com maior precisão a anatomia e função ventricular. Além disso, graças a técnicas qualitativas e quantitativas com avaliação de fluxo, a RMC pode complementar a avaliação de regurgitação valvar em casos onde há dúvida diagnóstica ${ }^{52}$. Nas situações de estenoses valvares, alguns estudos recentes vem demonstrando que a RMC apresenta alta acurácia na determinação da área valvar, através da planimetria, em comparação com demais métodos de referência e pode ser utilizada também em situações onde há dúvida diagnóstica ${ }^{53}$. De qualquer modo, durante a avaliação da função ventricular em quaisquer situações, a simples detecção da disfunção valvar pode ser caracterizada pela RMC, pelo menos de forma qualitativa (Tabela 7).

\section{Tabela 7 - Indicações de RMC na avaliação das doenças valvares}

$\begin{array}{lc}\text { Indicação } & \text { Classe } \\ \text { 1. Avaliação da anatomia função ventricular } & \text { I } \\ \text { 2. Quantificação da regurgitação } & \text { I } \\ \text { 3. Quantificação e planimetria de estenoses } & \text { IIb } \\ \text { 4. Avaliação de próteses valvares } & \text { III } \\ \text { 5. Morfologia valvar } & \text { III } \\ \text { Valva aórtica bicúspide } & \text { III } \\ \text { Demais valvas } & \text { III } \\ \text { Vegetações } & \text { III }\end{array}$

\subsection{Análise de Custo-efetividade}

A análise de custo-efetividade de qualquer novo método diagnóstico é de fundamental importância, dada a limitação de recursos na área da saúde. A comparação de custos deve ser feita pelo médico que solicitar o exame sob três aspectos ${ }^{54}$ : a qualidade da informação obtida em termos de volume de informações e acurácia diagnóstica (evitando novos exames, por exemplo); riscos relacionados ao procedimento, evitando-se complicações onerosas destes; custo financeiro direto, sendo que, neste caso, o valor da RMC contrastada com gadolínio deve ser considerado semelhante ao custo de uma cintilografia de perfusão miocárdica por medicina nuclear.

\section{Tomografia Computadorizada}

O uso da tomografia computadorizada (TC) na área cardíaca vem ganhando grande destaque com a maior disponibilização de aparelhos de múltiplas colunas de detectores (TCMD - na literatura em inglês usa-se "multiple detector row", e também a denominação multislice $)^{55}$. 0 uso da TCMD na cardiologia se dá na determinação do escore de cálcio, método já utilizado há algum tempo, e, mais recentemente, na realização da angiotomografia coronária para avaliação da anatomia e de estenoses coronárias. 0 escore de cálcio é calculado por software específico e praticamente não é operador dependente, porém a angiotomografia coronária requer a participação intensa do médico na preparação do paciente, na aquisição, no pós-processamento e na interpretação das imagens.

\section{TÉCNICA, RADIAÇÃo E CONTRASTE NA TOMOGRAFIA}

A TC utiliza emissões de feixes de raio- $X$ que cruzam o corpo do indivíduo. A mesa no qual repousa o paciente pode estar parada (aquisição convencional, usada na aquisição do escore de cálcio) ou movimentando-se lentamente (aquisição helicoidal, usada na angiotomografia coronária). Os raios-X são captados por múltiplas colunas de detectores no lado oposto da abertura do gerador. Isto gera dados que são reconstruídos em imagens axiais do coração do paciente ${ }^{56}$.

As imagens digitais apresentam variações de atenuações, conforme a densidade dos tecidos, sendo medidas em unidades denominadas Hounsfield (HU). $\mathrm{Na}$ cardiologia, utilizava-se, até a introdução dos novos aparelhos de TCMD, tomógrafos de emissão de feixes de elétrons (EBCT), que tinham como principal objetivo a determinação do escore de cálcio. Atualmente, estes aparelhos vem sendo substituídos pelos aparelhos de TCMD, que apresentam como característica principal o maior número de detectores (4 a 64) na captação do feixe de raio- $X$. Estes equipamentos podem gerar imagens de alta resolução espacial (voxel de até 0,3 $\mathrm{mm}$ ) e também de alta resolução temporal (100 a 165 ms), devido a alta velocidade de rotação.

A TCMD é realizada com pausa respiratória que varia de 8 a 20 segundos, dependendo do número de 
detectores do tomógrafo, sendo a aquisição de imagem acoplada ao eletrocardiograma. Para sua realização, é fundamental que o ritmo seja regular, preferencialmente com freqüência cardíaca próxima a 60 bpm, sendo muitas vezes necessário o emprego de beta-bloqueadores orais ou endovenosos para se atingir esta freqüência. Para os exames de cálcio escore não é necessário a injeção de contraste, porém na angiografia coronária por TCMD a injeção de contraste iodado é fundamental (vide abaixo).

\section{Radiação}

Como a TC é baseada na emissão de raios- $X$, a preocupação com a exposição do paciente à radiação ionizante deve ser lembrada. A tabela 8 mostra a incidência de radiação na comparação de diversos métodos de imagem em cardiologia. Observa-se que a exposição da angiotomografia coronária é atualmente equivalente à da cintilografia para pesquisa de isquemia, podendo ser superior a um cateterismo diagnóstico ${ }^{57}$. Algumas técnicas de modulação de dose em TCMD podem ser empregadas atualmente, reduzindo a dose aplicada em até $40 \%$ às doses usuais.

\section{Contraste}

Os contrastes utilizados nos exames de angiotomografia coronária são a base de iodo e, diferentemente do gadolínio utilizado na RMC, possuem nefrotoxicidade e maior incidência de reações adversas. Deve-se dar preferência nos exames de angiotomografia coronária para contrastes não-iônicos com baixa ou iso-osmolalidade para redução destas reações adversas. Apesar disso, reações graves são relativamente raras ${ }^{58}$ e a nefrotoxicidade do contraste usualmente é auto-limitada, podendo ser prevenida através da boa hidratação do paciente anteriormente ao

Tabela 8 - Radiação dos métodos de tomografia e demais métodos diagnósticos em cardiologia ${ }^{57}$

Situação

Exposição natural ao ambiente (anual)

Radiografia de tórax - 2 incidências

Escore de cálcio (EBTC)

Escore de cálcio (TCMD)

Angio-TC (EBTC)

Angio-TC (TCMD) 16 detectores

Angio-TC (TCMD) 64 detectores

Angio-TC (TCMD) 16 detectores com

modulação de dose

Angio-TC (TCMD) 64 detectores com

modulação de dose

SPECT repouso + stress ( ${ }^{99} \mathrm{TC}$-sestamibi)

SPECT repouso $\left.{ }^{201} \mathrm{TI}\right)$

Angiografia coronária diagnóstica

Angioplastia coronária terapêutica

EBTC, tomografia computadorizada por emissão de elétrons;

TCMD, tomografia computadorizada de múltiplos detectores; $m S v$ milisievert; Tc, tecnésio; TI, tálio

* Média homens e mulheres exame. Em comparação à angiografia invasiva que utiliza uma média de $150 \mathrm{ml}$ de contraste, na angiotomografia de 80 a $120 \mathrm{ml}$ usualmente são utilizados.

\section{EsCORE DE CÁLCIO}

O escore de cálcio quantifica a calcificação arterial coronária (CAC), um marcador da presença e extensão da doença aterosclerótica. A calcificação é definida como uma lesão acima de $130 \mathrm{HU}$ e pode ser calculada a partir da soma ponderada das densidades acima de $130 \mathrm{HU}$ (escore de Agatston) ou por métodos de cálculo do volume ou massa de cálcio.

A avaliação do escore de cálcio adiciona informações no diagnóstico da DAC, complementando outras informações de fatores de risco clínico, podendo alterar e/ou acrescentar condutas, principalmente em pacientes classificados como risco intermediário pelo escore de Framingham ${ }^{59}$. Este fato já foi também validado na população brasileira onde mostrou-se a mesma importância clínica ${ }^{60}$. Assim, aceita-se que o escore de cálcio traga as seguintes informações:

1 ) escore de cálcio negativo $(C A C=0)$ indica uma baixa probabilidade de DAC e de eventos cardiovasculares futuros;

2) ausência de CAC é preditiva de baixo risco em um período de 2-5 anos;

3) escore de cálcio positivo $(C A C>0)$ confirma a presença de DAC;

4) valor de escore de cálcio alto ( $>400$ ou $>$ percentil 75 para a idade e sexo) significa risco moderado a alto de eventos clínicos em 2-5 anos;

5) medida da CAC é preditora independente de eventos e acrescenta valor prognóstico em relação aos fatores de risco tradicionais de Framingham e à proteína C-reativa;

6) a quantificação da CAC pode alterar a conduta clínica, principalmente em pacientes de risco intermediário.

O escore de cálcio tem suas limitações e não deve ser utilizado para estimar a presença de obstruções luminais, não deve ser utilizado para monitoramento de resposta terapêutica e não deve ser utilizado na fase aguda ou em pacientes que já tenham documentação de DAC comprovada ${ }^{61}$. As recomendações para uso do escore de cálcio estão listadas na Tabela 9.

\section{Angiotomografia de coronárias}

O uso da TCMD para realização da angiotomografia de coronárias é hoje uma realidade clínica62, aplicável em casos específicos conforme segue abaixo. A realização de angiografia coronária não invasiva é factível para aparelhos com capacidade de adquirir, no mínimo, 16 cortes por rotação. 
Tabela 9 - Indicações de realização de TCMD para avaliação do escore de cálcio

Indicação
1. Pacientes assintomáticos com risco intermediário
de eventos ( $10-20 \%$ em 10 anos) pelos critérios de
Framingham
2. Pacientes assintomáticos com histórico familiar de
DAC precoce.
3. Pacientes de baixo risco pelo escore de Framingham
(< $10 \%$ em 10 anos)
4. Pacientes de alto risco pelo escore de Framingham
(>20\% em 10 anos) ou com doença arterial coronária
já diagnosticada
5. Seguimento da evolução do escore de cálcio
DAC- doença arterial coronária

\section{Artérias coronárias nativas}

A realização da angiotomografia de coronárias está sendo alvo de extensa pesquisa, com grande repercussão nas principais revistas médicas ${ }^{63}$. Os principais trabalhos com aparelhos de 16 e 64 cortes por rotação estão apresentados na Tabela $10^{64,65}$. Os aparelhos de 64 detectores permitiram ganho marginal de resolução espacial, mas uma redução de cerca de $40 \%$ no tempo de apnéia, reduzindo artefatos respiratórios e de variações do ritmo cardíaco, aumentando o número de artérias analisáveis.

A experiência com angiotomografia de coronárias, entretanto, ainda é limitada a populações específicas, não sendo ainda estudado seu papel em comparação com outros métodos não invasivos de detecção de DAC ou em pacientes com baixo risco para a doença. Além disso, usualmente foram estudados apenas artérias com calibre superior a $1,5 \mathrm{~mm}$, não sendo clinicamente viável na rotina a aplicação do método para análise quantitativa de estenoses arteriais.

Assim, o método tem maior aplicação em pacientes com probabilidade intermediária de DAC e testes funcionais não diagnósticos ou conflitantes ou em pacientes com baixa probabilidade com testes funcionais positivos $^{66}$. 0 exame não deve ser realizado ainda para pacientes com baixa probabilidade de DAC assintomáticos ou com teste de isquemia negativos ou para seguimento de lesões obstrutivas identificadas em angiografia prévia. Além disso, grande quantidade de cálcio coronário pode limitar a interpretação completa do exame. Um resumo das indicações atuais da angiotomografia de coronária está na tabela 11 .

\section{Re-estenose intra-stent}

A angiotomografia de coronárias para avaliação da re-estenose intra-stent não deve ser utilizada na rotina clínica de maneira geral, pois tem sensibilidade apenas moderada ${ }^{67}$. Porém, quando são analisados apenas stents com diâmetros superiores a $3 \mathrm{~mm}$ ou aqueles implantados em tronco de coronária esquerda, a acurácia diagnóstica aumenta significativamente ${ }^{68}$.

\section{Enxertos coronários cirúrgicos}

A determinação da patência de enxertos coronários pela TCMD tem alta acurácia diagnóstica ${ }^{69}$, sendo limitada apenas por artefatos dos clipes cirúrgicos e menor sensibilidade diagnóstica dos trechos distais das anastomoses ou grande quantidade de cálcio em pacientes com DAC avançada.

\section{Angiotomografia na avaliação da dor torácica}

O uso da angio-TC em situações de dor torácica aguda ainda tem experiência limitada mas recentes estudos demonstraram alta sensibilidade e especificidade no diagnóstico de DAC nestas situações ${ }^{70}$. Além disso, com protocolos específicos ${ }^{71}$, pode-se fazer o diagnóstico diferencial de síndrome coronariana aguda, dissecção de aorta e embolia pulmonar ("descarte triplo").

Tabela 10 - Desempenho diagnóstico da TCMD na detecção de estenoses coronárias em artérias nativas

\begin{tabular}{|c|c|c|c|c|c|c|c|c|c|}
\hline Estudo & $\begin{array}{l}\text { Cortes por } \\
\text { rotação }\end{array}$ & $\mathbf{n}$ & Vasos & Risco de DAC & $\begin{array}{l}\text { Exclusão } \\
(\%)\end{array}$ & Sens. & Especif. & VPP & VPN \\
\hline Nieman $2002^{76}$ & 12 & 58 & $>2.0 \mathrm{~mm}$ & Alto & - & 95 & 86 & 80 & 97 \\
\hline Ropers $2003^{64 *}$ & 12 & 77 & $>1.5 \mathrm{~mm}$ & Alto & 12 & 92 & 93 & 79 & 97 \\
\hline Hoffmann $2004^{77}$ & 16 & 33 & segmento & Alto & 17 & 63 & 95 & 64 & 96 \\
\hline Mollet $2004^{78 *}$ & 16 & 128 & $>2.0 \mathrm{~mm}$ & Baixo-mod. & - & 92 & 95 & 79 & 98 \\
\hline Martuscelli $2004^{79}$ & 16 & 64 & $>1.5 \mathrm{~mm}$ & Mod. & 16 & 89 & 98 & 90 & 98 \\
\hline Hoffmann $2005^{63}$ & 16 & 103 & $>1.5 \mathrm{~mm}$ & Mod-alto & 6.4 & 97 & 87 & 90 & 95 \\
\hline Kefer $2005^{34}$ & 16 & 52 & $>1.5 \mathrm{~mm}$ & Mod & - & 82 & 79 & 56 & 83 \\
\hline Kuettner $2005^{80}$ & 16 & 120 & segmento & Mod-alto & - & 85 & 98 & 91 & 96 \\
\hline Mollet $2005^{65 *}$ & 64 & 52 & segmento & Alto & - & 99 & 95 & 76 & 99 \\
\hline Leber $2005^{81}$ & 64 & 55 & segmento & Mod-alto & 7 & 73 & 97 & 75 & 97 \\
\hline Raff 2005 & 64 & 70 & segmento & Alto & 12 & 95 & 90 & 93 & 93 \\
\hline Ropers $2006^{83 *}$ & 64 & 84 & $>1.5 \mathrm{~mm}$ & Alto & 4 & 96 & 91 & 76 & 100 \\
\hline
\end{tabular}




\begin{tabular}{|c|c|}
\hline Indicação & Classe \\
\hline 1. Avaliação de coronárias anômalas & 1 \\
\hline $\begin{array}{l}\text { 2. Avaliação de estenoses coronárias em pacientes com } \\
\text { probabilidade intermediária de DAC e testes de isquemia } \\
\text { duvidosos ou conflitantes }\end{array}$ & Ila \\
\hline $\begin{array}{l}\text { 3. Avaliação de estenoses coronárias em pacientes } \\
\text { com baixa probabilidade de DAC e testes de isquemia } \\
\text { positivos }\end{array}$ & Ila \\
\hline 4. Avaliação da patência de enxertos cirúrgicos & Ila \\
\hline $\begin{array}{l}\text { 5. Opção à angiografia invasiva no diagnóstico } \\
\text { diferencial de cardiomiopatias isquêmicas versus não } \\
\text { isquêmicas }\end{array}$ & Ila \\
\hline $\begin{array}{l}\text { 6. Opção à angiografia invasiva no seguimento de } \\
\text { pacientes com doença de Kawasaki }\end{array}$ & Ila \\
\hline $\begin{array}{l}\text { 7. Diagnóstico de estenoses coronárias em pacientes } \\
\text { com média/alta probabilidade de DAC com dor torácica } \\
\text { aguda }\end{array}$ & $\mathrm{Ilb}$ \\
\hline $\begin{array}{l}\text { 8. Diagnóstico de estenoses intra-stents (vide } \\
\text { observações no texto) }\end{array}$ & $\mathrm{Ilb}$ \\
\hline $\begin{array}{l}\text { 9. Pacientes com baixa probabilidade de DAC, } \\
\text { assintomáticos e/ou com teste de isquemia negativo }\end{array}$ & III \\
\hline $\begin{array}{l}\text { 10. Seguimento de lesões obstrutivas coronárias } \\
\text { identificadas em angiografia prévia (invasiva ou não } \\
\text { invasiva). }\end{array}$ & III \\
\hline DAC- doença arterial coronária. & \\
\hline
\end{tabular}

\section{Doenças Vasculares}

A avaliação de doenças vasculares pela angiotomografia apresenta aplicação clínica consagrada, se destacando ainda mais recentemente com a tecnologia da TCMD. Exames de toda a aorta torácica e abdominal podem

\section{Tabela 12 - Indicações de TCMD na avaliação das doenças vasculares}

Indicação Classe

1. Aneurismas de aorta (incluindo Marfan)

2. Dissecção de aorta (aguda e crônica)

3. Ruptura de aorta

4. Hematoma intramural aórtico

5. Úlceras aórticas

6. Planejamento de abordagem cirúrgica da aorta

7. Planejamento de stent aórtico

8. Avaliação pós operatória de implantes de stents

9. Anatomia da artéria pulmonar

10. Embolia Pulmonar

11. Avaliação das veias pulmonares

12. Avaliação estenoses renais

13. Avaliação de estenoses carótidas extra-cranianas

14. Avaliação das artérias mesentéricas e tronco celíaco

15. Arterites

Classe

ser realizados em menos de 20 segundos, com alta sensibilidade e especificidade ${ }^{72}$, podendo, inclusive, se realizar a avaliação de endopróteses.

Além da aplicação nas doenças da aorta, na avaliação pulmonar a TCMD permite o diagnóstico de quadros de embolia pulmonar ${ }^{73}$, auxiliando, graças ao amplo campo visual, no diagnóstico diferencial com outras doenças pulmonares. A avaliação das veias pulmonares também é possível pela angiotomografia de forma semelhante à da RMC.

Finalmente, a angiotomografia tem alta sensibilidade e especificidade para detecção de obstruções das carótidas $^{74}$, podendo também ser utilizada para diagnóstico de alterações de vasos mesentéricos e na avaliação de estenoses de artérias renais ${ }^{75}$ (Tabela 12 ).

\section{REFERÊNCIAS}

1. O'Rourke RA, Brundage BH, Froelicher VF, Greenland P, Grundy SM, Hachamovitch R, et al. American College of Cardiology/American Heart Association Expert Consensus Document on electron-beam computed tomography for the diagnosis and prognosis of coronary artery disease. J Am Coll Cardiol 2000;36:326-340.

2. Pennell DJ, Sechtem UP, Higgins CB, Manning WJ, Pohost GM, Rademakers FE, et al. Clinical indications for cardiovascular magnetic resonance (CMR): Consensus Panel report. Eur Heart J 2004;25:1940-65.

3. Gluud C, Gluud LL. Evidence based diagnostics. Bmj 2005;330:724-

4. Gerber TC, Fasseas P, Lennon RJ, Valeti VU, Wood CP, Breen JF, et al. Clinical safety of magnetic resonance imaging early after coronary artery stent placement. J Am Coll Cardiol 2003;42:1295-8.

5. Schneider G, Fries P, Ahlhelm F, Kindermann I, Kramann B, Bohm M. Contrast-enhanced cardiac MR imaging. Eur Radiol 2003;13 Suppl 3:N11-8.

6. Kirchin MA, Runge VM. Contrast agents for magnetic resonance imaging: safety update. Top Magn Reson Imaging 2003;14:42635.

7. Tsai-Goodman B, Geva T, Odegard KC, Sena LM, Powell AJ. Clinical role, accuracy, and technical aspects of cardiovascular magnetic resonance imaging in infants. Am J Cardiol 2004;94:69-74.

8. Hoppe UC, Dederichs B, Deutsch HJ, Theissen P, Schicha H, Sechtem $U$. Congenital heart disease in adults and adolescents: comparative value of transthoracic and transesophageal echocardiography and MR imaging. Radiology 1996;199:669-677.

9. Geva T, Vick GW, III, Wendt RE, Rokey R. Role of spin echo and cine magnetic resonance imaging in presurgical planning of heterotaxy syndrome. Comparison with echocardiography and catheterization. Circulation 1994;90:348-356.

10. Kersting-Sommerhoff BA, Diethelm L, Stanger P, Dery R, Higashino SM, Higgins SS, et al. Evaluation of complex congenital ventricular anomalies with magnetic resonance imaging. Am. Heart J. 1990;120:133-142.

11. White CS, Baffa JM, Haney PJ, Pace ME, Campbell AB. MR imaging of congenital anomalies of the thoracic veins. Radiographics 1997; 17:595-608.

12. Dill T, Neumann T, Ekinci O, Breidenbach C, John A, Erdogan A, et al. Pulmonary vein diameter reduction after radiofrequency catheter ablation for paroxysmal atrial fibrillation evaluated by contrastenhanced three-dimensional magnetic resonance imaging. Circulation 2003; 107:845-850. 
13. Bogaert J, Kuzo R, Dymarkowski S, Janssen L, Celis I, Budts W, et al. Follow-up of patients with previous treatment for coarctation of the thoracic aorta: comparison between contrast-enhanced MR angiography and fast spin-echo MR imaging. Eur.Radiol. 2000;10:1847-1854.

14. Niezen RA, Helbing WA, van der Wall EE, van der Geest RJ, Rebergen SA, de Roos A. Biventricular systolic function and mass studied with MR imaging in children with pulmonary regurgitation after repair for tetralogy of Fallot. Radiology 1996;201:135-140.

15. Greil GF, Stuber M, Botnar RM, Kissinger KV, Geva T, Newburger JW, et al. Coronary magnetic resonance angiography in adolescents and young adults with kawasaki disease. Circulation 2002;105:908911.

16. Joarder R, Gedroyc WM. Magnetic resonance angiography: the state of the art. Eur Radiol 2001;11:446-53.

17. Goldman JP. New techniques and applications for magnetic resonance angiography. Mt Sinai J Med 2003;70:375-85.

18. Leiner T, de Haan MW, Nelemans PJ, van Engelshoven JM, Vasbinder GB. Contemporary imaging techniques for the diagnosis of renal artery stenosis. Eur Radiol 2005;15:2219-29.

19. Sensky PR, Jivan A, Hudson NM, Keal RP, Morgan B, Tranter JL, et al. Coronary artery disease: combined stress MR imaging protocolone-stop evaluation of myocardial perfusion and function. Radiology 2000;215:608-14

20. Chuang ML, Hibberd MG, Salton CJ, Beaudin RA, Riley MF, Parker $R A$, et al. Importance of imaging method over imaging modality in noninvasive determination of left ventricular volumes and ejection fraction: assessment by two- and three-dimensional echocardiography and magnetic resonance imaging. J Am Coll Cardiol 2000;35:47784.

21. Nagel E, Lorenz C, Baer F, Hundley WG, Wilke N, Neubauer S, et al. Stress cardiovascular magnetic resonance: consensus panel report. J.Cardiovasc. Magn Reson. 2001;3:267-281.

22. Hundley WG, Morgan TM, Neagle CM, Hamilton CA, Rerkpattanapipat $\mathrm{P}$, Link KM. Magnetic resonance imaging determination of cardiac prognosis. Circulation 2002;106:2328-2333.

23. Rerkpattanapipat P, Morgan TM, Neagle CM, Link KM, Hamilton CA, Hundley WG. Assessment of preoperative cardiac risk with magnetic resonance imaging. Am.J.Cardiol. 2002;90:416-419.

24. Giang TH, Nanz D, Coulden R, Friedrich M, Graves M, al Saadi N, et al. Detection of coronary artery disease by magnetic resonance myocardial perfusion imaging with various contrast medium doses: first European multi-centre experience. Eur. Heart J. 2004;25:16571665.

25. Panting JR, Gatehouse PD, Yang GZ, Jerosch-Herold M, Wilke $\mathrm{N}$, Firmin DN, et al. Echo-planar magnetic resonance myocardial perfusion imaging: parametric map analysis and comparison with thallium SPECT. J.Magn Reson. Imaging 2001;13:192-200.

26. Lee DC, Simonetti OP, Harris KR, Holly TA, Judd RM, Wu E, et al. Magnetic resonance versus radionuclide pharmacological stress perfusion imaging for flow-limiting stenoses of varying severity. Circulation 2004; 110:58-65.

27. Ingkanisorn WP, Kwong RY, Bohme NS, Geller NL, Rhoads KL, Dyke CK, et al. Prognosis of Negative Adenosine Stress Magnetic Resonance in Patients Presenting to an Emergency Department With Chest Pain. J Am Coll Cardiol 2006;47:1427-32.

28. Simonetti OP, Kim RJ, Fieno DS, Hillenbrand HB, Wu E, Bundy JM, et al. An improved MR imaging technique for the visualization of myocardial infarction. Radiology 2001;218:215-223.

29. Wagner A, Mahrholdt H, Holly TA, Elliott MD, Regenfus M, Parker $\mathrm{M}$, et al. Contrast-enhanced MRI and routine single photon emission computed tomography (SPECT) perfusion imaging for detection of subendocardial myocardial infarcts: an imaging study. Lancet 2003;361:374-379.

30. Azevedo Filho CF, Hadlich M, Petriz JL, Mendonca LA, Moll Filho J, Rochitte CE. Quantification of left ventricular infarcted mass on cardiac magnetic resonance imaging: comparison between planimetry and the semiquantitative visual scoring method. Arq Bras Cardiol 2004;83:118-24; 111-7.

31. Gerber BL, Garot J, Bluemke DA, Wu KC, Lima JA. Accuracy of contrast-enhanced magnetic resonance imaging in predicting improvement of regional myocardial function in patients after acute myocardial infarction. Circulation 2002;106:1083-1089.

32. Rochitte CE, Lima JA, Bluemke DA, Reeder SB, McVeigh ER, Furuta T, et al. Magnitude and time course of microvascular obstruction and tissue injury after acute myocardial infarction. Circulation 1998;98:1006-1014.

33. Kim RJ, Wu E, Rafael A, Chen EL, Parker MA, Simonetti O, et al. The use of contrast-enhanced magnetic resonance imaging to identify reversible myocardial dysfunction. N.Engl.J.Med. 2000;343:1445 1453

34. Kefer J, Coche E, Legros G, Pasquet A, Grandin C, Van Beers BE, et al. Head-to-head comparison of three-dimensional navigator-gated magnetic resonance imaging and 16-slice computed tomography to detect coronary artery stenosis in patients. J Am Coll Cardiol 2005;46:92-100.

35. Moon JC, Fisher NG, McKenna WJ, Pennell DJ. Detection of apica hypertrophic cardiomyopathy by cardiovascular magnetic resonance in patients with non-diagnostic echocardiography. Heart 2004;90:6459.

36. Moon JC, McKenna WJ, McCrohon JA, Elliott PM, Smith GC, Pennel DJ. Toward clinical risk assessment in hypertrophic cardiomyopathy with gadolinium cardiovascular magnetic resonance. J Am Coll Cardiol 2003;41:1561-7

37. Petersen SE, Selvanayagam JB, Francis JM, Myerson SG, Wiesmann $\mathrm{F}$, Robson MD, et al. Differentiation of athlete's heart from pathological forms of cardiac hypertrophy by means of geometric indices derived from cardiovascular magnetic resonance. J Cardiovasc Magn Reson $2005 ; 7: 551-8$

38. Soriano CJ, Ridocci F, Estornell J, Jimenez J, Martinez V, De Velasco $J A$. Noninvasive diagnosis of coronary artery disease in patients with heart failure and systolic dysfunction of uncertain etiology, using late gadolinium-enhanced cardiovascular magnetic resonance. J.Am.Coll. Cardiol. 2005;45:743-748.

39. Rochitte CE, Oliveira PF, Andrade JM, Ianni BM, Parga JR, Avila LF, et al. Myocardial delayed enhancement by magnetic resonance imaging in patients with Chagas' disease: a marker of disease severity. J Am Coll Cardiol 2005;46:1553-8.

40. Keller DI, Osswald S, Bremerich J, Bongartz G, Cron TA, Hilti P, et al. Arrhythmogenic right ventricular cardiomyopathy: diagnostic and prognostic value of the cardiac MRI in relation to arrhythmia-free survival. Int.J.Cardiovasc. Imaging 2003;19:537-543.

41. Anderson LJ, Holden S, Davis B, Prescott E, Charrier CC, Bunce NH, et al. Cardiovascular T2-star (T2*) magnetic resonance for the early diagnosis of myocardial iron overload. Eur. Heart J. 2001;22:2171 2179.

42. Sechtem U, Higgins CB, Sommerhoff BA, Lipton MJ, Huycke EC. Magnetic resonance imaging of restrictive cardiomyopathy. Am.J.Cardiol. 1987;59:480-482.

43. Abdel-Aty H, Boye P, Zagrosek A, Wassmuth R, Kumar A, Messroghli $D$, et al. Diagnostic performance of cardiovascular magnetic resonance in patients with suspected acute myocarditis: comparison of different approaches. J Am Coll Cardiol 2005;45:1815-22.

44. Magnani JW, Dec GW. Myocarditis: current trends in diagnosis and treatment. Circulation 2006;113:876-90.

45. Varghese A, Fisher NG, Pennell DJ. Late recognition of left ventricular non-compaction by cardiovascular magnetic resonance. Heart 2005;91:282.

46. Petersen SE, Selvanayagam JB, Wiesmann F, Robson MD, Francis $\mathrm{JM}$, Anderson $\mathrm{RH}$, et al. Left ventricular non-compaction: insights from cardiovascular magnetic resonance imaging. J Am Coll Cardiol 2005;46:101-5. 
47. Smedema JP, Snoep G, van Kroonenburgh MP, van Geuns RJ, Dassen WR, Gorgels AP, et al. Evaluation of the accuracy of gadoliniumenhanced cardiovascular magnetic resonance in the diagnosis of cardiac sarcoidosis. J.Am.Coll.Cardiol. 2005;45:1683-1690.

48. Francone M, Dymarkowski S, Kalantzi M, Rademakers FE, Bogaert $\mathrm{J}$. Assessment of ventricular coupling with real-time cine MRI and its value to differentiate constrictive pericarditis from restrictive cardiomyopathy. Eur Radiol 2005:1-8.

49. Frank H, Globits S. Magnetic resonance imaging evaluation of myocardial and pericardial disease. J Magn Reson Imaging 1999;10:617-26.

50. Hoffmann U, Globits S, Schima W, Loewe C, Puig S, Oberhuber G, et al. Usefulness of magnetic resonance imaging of cardiac and paracardiac masses. Am J Cardiol 2003;92:890-5.

51. Barkhausen J, Hunold P, Eggebrecht H, Schuler WO, Sabin GV, Erbel $\mathrm{R}$, et al. Detection and characterization of intracardiac thrombi on MR imaging. AJR Am J Roentgenol 2002;179:1539-44.

52. Krombach GA, Kuhl H, Bucker A, Mahnken AH, Spuntrup E, Lipke C, et al. Cine MR imaging of heart valve dysfunction with segmented true fast imaging with steady state free precession. J Magn Reson Imaging 2004; 19:59-67.

53. John AS, Dill T, Brandt RR, Rau M, Ricken W, Bachmann G, et al. Magnetic resonance to assess the aortic valve area in aortic stenosis: how does it compare to current diagnostic standards? J Am Coll Cardiol 2003;42:519-26.

54. Araujo DV, Ferraz MB. [Economic impact of chronic ischemic cardiopathy treatment in Brazil. The challenge of new cardiovascular technology inclusion]. Arq Bras Cardiol 2005;85:1-2.

55. Schoenhagen P, Halliburton SS, Stillman AE, Kuzmiak SA, Nissen SE, Tuzcu EM, et al. Noninvasive imaging of coronary arteries: current and future role of multi-detector row CT. Radiology 2004;232:7-17.

56. Flohr TG, Schaller S, Stierstorfer K, Bruder H, Ohnesorge BM, Schoepf UJ. Multi-detector row CT systems and image-reconstruction techniques. Radiology 2005;235:756-73.

57. Hausleiter J, Meyer T, Hadamitzky M, Huber E, Zankl M, Martinoff $\mathrm{S}$, et al. Radiation dose estimates from cardiac multislice computed tomography in daily practice: impact of different scanning protocols on effective dose estimates. Circulation 2006;113:1305-10.

58. Morcos SK, Thomsen HS. Adverse reactions to iodinated contrast media. Eur Radiol 2001;11:1267-75.

59. Greenland P, LaBree L, Azen SP, Doherty TM, Detrano RC. Coronary artery calcium score combined with Framingham score for risk prediction in asymptomatic individuals. JAMA 2004;291:210-215.

60. Meneghelo RS, Santos RD, Almeida B, Hidal J, Martinez T, Moron R, et al. Distribution of coronary artery calcium scores determined by ultrafast computed tomography in 2.253 asymptomatic white men. Arq Bras.Cardiol. 2003;81 Suppl 7:27-36.

61. Chen J, Krumholz HM. How useful is computed tomography for screening for coronary artery disease? Screening for coronary artery disease with electron-beam computed tomography is not useful. Circulation 2006;113:125-46; discussion 125-46.

62. Garcia MJ. Noninvasive coronary angiography: hype or new paradigm? Jama 2005;293:2531-3.

63. Hoffmann MH, Shi H, Schmitz BL, Schmid FT, Lieberknecht M, Schulze $\mathrm{R}$, et al. Noninvasive coronary angiography with multislice computed tomography. Jama 2005;293:2471-8

64. Ropers D, Baum U, Pohle K, Anders K, Ulzheimer S, Ohnesorge B, et al. Detection of coronary artery stenoses with thin-slice multi-detector row spiral computed tomography and multiplanar reconstruction. Circulation 2003;107:664-6.

65. Mollet NR, Cademartiri F, van Mieghem CA, Runza G, McFadden EP, Baks T, et al. High-resolution spiral computed tomography coronary angiography in patients referred for diagnostic conventional coronary angiography. Circulation 2005;112:2318-23.
66. Sechtem U, Vohringer M. The clinical role of 'non-invasive' coronary angiography by multidetector spiral computed tomography: yet to be defined. Eur Heart J 2005;26:1942-4.

67. Gaspar T, Halon DA, Lewis BS, Adawi S, Schliamser JE, Rubinshtein R, et al. Diagnosis of coronary in-stent restenosis with multidetector row spiral computed tomography. J Am Coll Cardiol 2005;46:1573-9.

68. Gilard M, Cornily JC, Pennec PY, Le Gal G, Nonent M, Mansourati $\mathrm{J}$, et al. Assessment of coronary artery stents by 16 slice computed tomography. Heart 2006;92:58-61.

69. Burgstahler C, Beck T, Kuettner A, Drosch T, Kopp AF, Heuschmid M, et al. Non-invasive evaluation of coronary artery bypass grafts using 16 -row multi-slice computed tomography with $188 \mathrm{~ms}$ temporal resolution. Int J Cardiol 2006;106:244-9.

70. Schroeder S, Kuettner A, Beck T, Kopp AF, Herdeg C, Heuschmid $M$, et al. Usefulness of noninvasive MSCT coronary angiography as first-line imaging technique in patients with chest pain: initial clinical experience. Int J Cardiol 2005;102:469-75.

71. Savino G, Herzog C, Costello P, Schoepf UJ. 64 slice cardiovascular CT in the emergency department: concepts and first experiences. Radiol Med (Torino) 2006;111:481-96.

72. Gotway MB, Dawn SK. Thoracic aorta imaging with multisclice CT. Radiol Clin North Am 2003;41:521-43.

73. Siegel MD. Multidetector-row computed tomography in suspected pulmonary embolism. N Engl J Med 2005;353:630-1; author reply 630-1.

74. Koelemay MJ, Nederkoorn PJ, Reitsma JB, Majoie CB. Systematic review of computed tomographic angiography for assessment of carotid artery disease. Stroke 2004;35:2306-12.

75. Weise WJ, Jaffrey JB. Review: CT angiography and magnetic resonance imaging are the best less-invasive tests for renal artery stenosis. ACP J Club 2002;136:69.

76. Nieman K, Cademartiri F, Lemos PA, Raaijmakers R, Pattynama PM, de Feyter PJ. Reliable noninvasive coronary angiography with fast submillimeter multislice spiral computed tomography. Circulation 2002;106:2051-4.

77. Hoffmann U, Moselewski F, Cury RC, Ferencik M, Jang IK, Diaz LJ, et al. Predictive value of 16-slice multidetector spiral computed tomography to detect significant obstructive coronary artery disease in patients at high risk for coronary artery disease: patient-versus segment-based analysis. Circulation 2004;110:2638-43.

78. Mollet NR, Cademartiri F, Krestin GP, McFadden EP, Arampatzis CA, Serruys PW, et al. Improved diagnostic accuracy with 16-row multislice computed tomography coronary angiography. J Am Coll Cardiol 2005;45:128-32.

79. Martuscelli E, Romagnoli A, D’Eliseo A, Razzini C, Tomassini M, Sperandio M, et al. Accuracy of thin-slice computed tomography in the detection of coronary stenoses. Eur Heart J 2004;25:1043-8.

80. Kuettner A, Beck T, Drosch T, Kettering K, Heuschmid M, Burgstahler $C$, et al. Diagnostic accuracy of noninvasive coronary imaging using 16-detector slice spiral computed tomography with 188 ms temporal resolution. J Am Coll Cardiol 2005;45:123-7.

81. Leber AW, Knez A, von Ziegler F, Becker A, Nikolaou K, Paul S, et al. Quantification of obstructive and nonobstructive coronary lesions by 64-slice computed tomography: a comparative study with quantitative coronary angiography and intravascular ultrasound. J Am Coll Cardiol 2005; 46:147-54.

82. Raff GL, Gallagher MJ, O'Neill WW, Goldstein JA. Diagnostic accuracy of noninvasive coronary angiography using 64-slice spiral computed tomography. J Am Coll Cardiol 2005;46:552-7.

83. Ropers D, Rixe J, Anders K, Kuttner A, Baum U, Bautz W, et al. Usefulness of multidetector row spiral computed tomography with 64- x 0.6-mm collimation and 330-ms rotation for the noninvasive detection of significant coronary artery stenoses. Am J Cardiol 2006;97:343-8. 